\title{
Physiological Responses to In-Bed Cycle Ergometry Treatment in Intensive Care Unit Patients with External Ventricular Drainage
}

\author{
Elizabeth K. Zink ${ }^{1,5^{*}}$, Sowmya Kumble ${ }^{2}$, Meghan Beier ${ }^{2}$, Pravin George ${ }^{3}$, Robert D. Stevens ${ }^{4,5}$ \\ and Mona N. Bahouth ${ }^{5}$
}

\begin{abstract}
Purpose: Evidence suggests that early physical activity can be accomplished safely in the neurocritical care unit (NCCU); however, many NCCU patients are often maintained in a state of inactivity due to impaired consciousness, sensorimotor deficits, and concerns for intracranial pressure elevation or cerebral hypoperfusion in the setting of autoregulatory failure. Structured in-bed mobility interventions have been proposed to prevent sequelae of complete immobility in such patients, yet the feasibility and safety of these interventions is unknown. We studied neurological and hemodynamic changes before and after cycle ergometry (CE) in a subset of NCCU patients with external ventricular drains (EVDs).

Methods: Patients admitted to the NCCU who had an EVD placed for cerebrospinal fluid drainage and intracranial pressure (ICP) monitoring underwent supine CE therapy with passive and active cycling settings. Neurologic status, ICP and hemodynamic parameters were monitored before and after each CE session.

Results: Twenty-seven patients successfully underwent in-bed CE in the NCCU. No clinically significant changes were recorded in neurologic or in physiological parameters before or after CE. There were no device dislodgements or other adverse effects requiring cessation of a CE session.

Conclusion: These data suggest that supine CE in a heterogeneous cohort of neurocritical care patients with EVDs is safe and tolerable. Larger prospective studies are needed to determine the efficacy and optimal dose and timing of supine CE in neurocritical care patients.
\end{abstract}

Keywords: Neurocritical care, External ventricular drain, Early mobility, Supine cycling, Recovery, Ergometry

\section{Introduction}

The benefits of early mobility in critically ill patients are widely recognized. However, implementation of such programs in the Neuro-Critical Care Unit (NCCU) presents unique challenges. This is particularly true for patients who: [1] have disorders of consciousness and sensorimotor impairments, [2] are at risk for intracranial

\footnotetext{
*Correspondence: ezink1@jhmi.edu

${ }^{1}$ Department of Neurosciences Nursing, The Johns Hopkins Hospital, 1800 Orleans Street, Zayed 3 West, Room 3074, Baltimore, MD 21287, USA Full list of author information is available at the end of the article
}

hypertension or cerebral ischemia, [3] have neurologic and hemodynamic instability, [4] are unable to tolerate interruptions in cerebrospinal fluid (CSF) drainage from an external ventricular drain (EVD), or [5] have a loss of calvarial integrity following decompressive craniectomy [1-3]. Since many such patients are prescribed strict bed rest, varied approaches are needed to combat the effects of immobility in this population. Supine cycle ergometry (CE) allows patients confined to a hospital bed or recliner chair to engage in repetitive active or passive exercises using their upper or lower extremities with the help of a built-in motor coupled with sensors that detect active 
motor activity in the extremities [4, 5]. Recent studies demonstrate that in-bed cycling is feasible and safe, and improves functional exercise capacity and isometric quadriceps force in medical and surgical ICU patients [6-8]. Additionally, early passive cycling within $72 \mathrm{~h}$ was found to be safe in a small population of sedated and mechanically ventilated patients even if they were receiving vasoactive agents $[9,10]$. Given its safety profile, ease of use, and positive outcomes in medical and surgical ICUs, in-bed supine CE may serve as a valuable modality for critically ill neurological patients. A recent study reported on the safety of passive supine $C E$ in patients with intracranial pressure monitoring and this report demonstrated feasibility in a small cohort [5]. However, little is known about the physiological responses to $\mathrm{CE}$ particularly in patients who are dependent on external CSF drainage. Here, we aim to evaluate the feasibility and safety of active and passive $\mathrm{CE}$ in a heterogeneous group of NCCU patients with EVDs.

\section{Methods}

We conducted a retrospective analysis of prospectively collected data as part of an ongoing Institutional Review Board-acknowledged quality improvement project between January 2015 and September 2016. Patients were included if they were admitted to the NCCU, received CE therapy and had an external ventricular drain (EVD) in place while cycling. Patients were eligible for cycling according to the unit-based mobility and activity algorithm if their motor sub-score on the Glasgow coma scale was less than 4 or they were specifically prescribed bed rest [2]. Patients were excluded from analysis if there was insufficient information collected about the type of session (active or passive) or duration of cycling and if they did not have an EVD. The presence of femoral catheters was not contraindicated in this study as previous studies of progressive mobility indicated that the benefits of mobility in critically ill patients outweighs the risks associated with these catheters $[11,12]$.

All procedures were conducted by a mobility team that included a physical therapist (PT) and a bedside NCCU nurse. Baseline neurological examination, intracranial pressure, cerebral perfusion pressure, mean arterial pressure, heart rate and pulse oximetry were recorded before and after each CE session as is the standard practice of physical therapists in this institution. This practice is based on published data indicating stability of vital signs, including intracranial pressure, before and after cycling sessions $[5,13]$.

Patients performed lower extremity CE exercise in the supine position with head of bed at 30 degrees, utilizing the MOTOmed Letto 2 (RECK-Technik GmbH \& Co, Germany, see Fig. 1). The EVD was clamped by the nurse if a change in the bed position was required for positioning the patient's legs in the CE, such that CSF could not inadvertently drain while the system was out of level. After the patient was positioned appropriately in the $\mathrm{CE}$, the EVD was leveled by the nurse and was left either clamped, precluding CSF drainage and facilitating ICP measurement or unclamped, facilitating CSF drainage, according to physician's orders. All ICP readings were performed with the EVD leveled at the external auditory meatus approximating the Foramen of Monro within the cerebral ventricular system. Patients performed either passive (cycle motor-initiated) or active (patient-initiated) supine cycling before and after their sessions based on their ability to participate and respond to therapist cueing. The cycle was set to a gear of 0 with the cycle providing at least 20 rotations per minute. The rotations per minute typically increase when the patient initiates

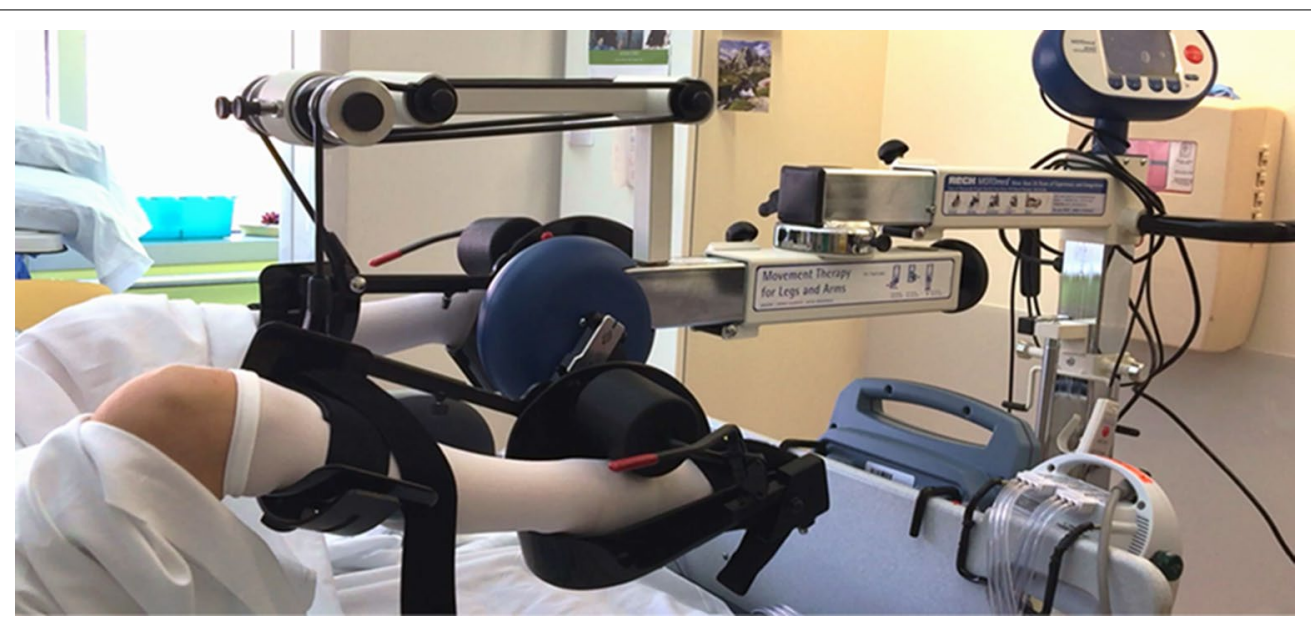

Fig. 1 Cycle ergometry of lower extremities Photo credit: P. George with permission 
cycling. The physical therapist or nurse remained at the bedside for the duration of the cycling session. Sessions were started only if a patient's baseline hemodynamic parameters fell within prescribed goals set by the medical team daily. The CE session was stopped immediately if a patient's vital signs fell outside of prescribed goals, if there was any evidence of neurologic deterioration, pain was unrelieved with repositioning in the cycle, or there was medical device dislodgement.

\section{Measures}

Physiological variables (blood pressure, mean arterial pressure (MAP), cerebral perfusion pressure (CPP), heart rate $(\mathrm{HR})$, intracranial pressure (ICP), pulse oximetry, and Glasgow Coma Scale were recorded immediately before and after the supine CE session. Safety thresholds were defined for each patient according to the individualized parameters based on underlying etiology of critical illness and set by the primary clinical team who were blinded to the goals of this study. Some patients received more than one supine CE session; however, only the first session was included in the analysis. Any adverse response that occurred during the session such as change in neurological function or device dislodgement as well as patient requesting to stop the cycling session were recorded. If patients were on a continuous infusion such as sedation, this information was also recorded. Cycling parameters were collected from CE screen and included total duration of the time spent on active and/or passive cycling (in minutes). If the patient performed active cycling, then the gear/resistance at which it was performed was also collected. Data were abstracted from physical therapist, nursing and provider notes in the electronic health record.

\section{Statistical Analysis}

Descriptive statistics were used to report demographic variables and session characteristics among participants who participated in the session actively or passively. Categorical variables were reported as counts and percentages. Continuous variables were reported as means with standard deviation. Only the first session per patient was analyzed. The active and passive CE session duration variables were set to zero if the patient spent less than one minute in the mode. Physiological variables were compared using paired t-tests. P-values $<0.05$ were considered statistically significant. Statistical analysis was performed using IBM $^{\circledR}$ SPSS, 2017.

\section{Results}

Graphs (histograms and Q-Q plots), as well as Kolmogorov-Smirnov and Shapiro-Wilk tests confirmed the physiologic variables were normally distributed. A

\section{Table 1 Patient characteristics}

\begin{tabular}{ll}
\hline Parameter & Patients, $\boldsymbol{n = 2 7}$ \\
\hline Age mean (SD) & $56(1305)$ \\
Sex \% male & 5506 \\
Diagnoses (\%) & \\
Subarachnoid hemorrhage & $10(37)$ \\
Intracerebral hemorrhage, infratentorial & $2(7.4)$ \\
\hline Intracerebral hemorrhage, supratentorial & $3(11.4)$ \\
Central nervous system infection & $5(18.5)$ \\
Ischemic stroke, posterior circulation & $2(7.4)$ \\
Hydrocephalus & $2(7.4)$ \\
\hline Brain tumor & $3(11.1)$ \\
Admission Glasgow coma scale IQR (median) & $7(10)$ \\
\hline Mechanical ventilation (\%) & $8(829.6)$ \\
Receiving continuous sedation (\%) & $3(11.1)$ \\
\hline
\end{tabular}

Raw scores are listed first

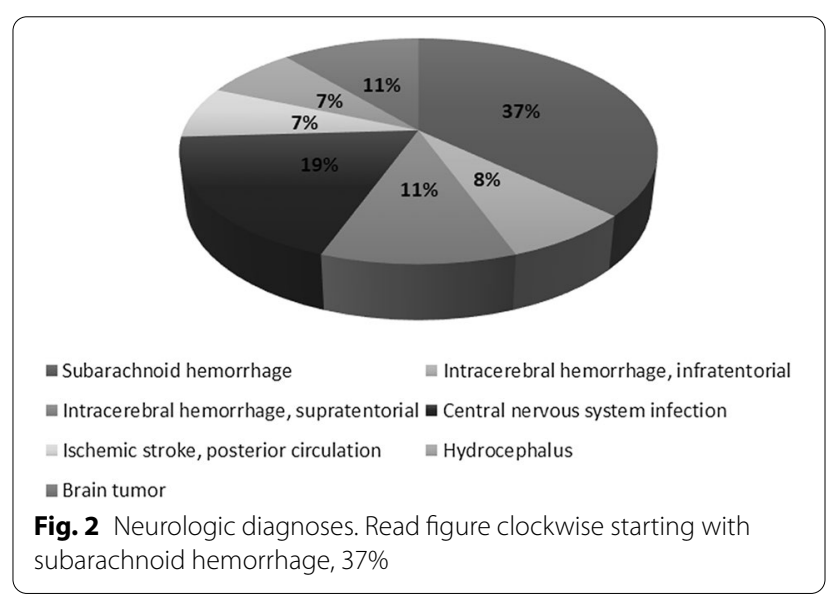

single outlier was observed for both the ICP and CPP measurements; therefore $\mathrm{t}$-tests were run with and without the outlier. The outlier did not have a significant influence over the results; therefore we retained all data for the final analyses.

Demographic features of the sample are provided in Table 1 and Fig. 2. Twenty-seven patients underwent at least one $\mathrm{CE}$ session for an average duration of $15 \mathrm{~min}$ (SD 4.6) with a minimum cycle time of $1 \mathrm{~min}$ and maximum cycle time of 25 . More than half of the CE sessions were conducted using a combination of passive and active cycling (Table 2). No device dislodgements or complications requiring treatment were noted during any of the sessions. Two patients experienced an increase in ICP above their prescribed target after the cycling session ended, the ICP returned to the prescribed target range without intervention and without a change in neurologic status. 
Table 2 Characteristics of cycling sessions

\begin{tabular}{ll}
\hline Parameter & Sessions, $\boldsymbol{n = 2 7}$ \\
\hline Cycling sessions & \\
Active only (\%) & $3(11.1)$ \\
\hline Passive only (\%) & $14(51.9)$ \\
Active and passive (\%) & $9(33.3)$ \\
\hline Active duration (min) (SD, minimum, maximum) & $5(6,3.5-15$ min) \\
Passive duration (min) (SD, minimum, maximum) & $10.0(8.2,1-25$ min)
\end{tabular}

Raw scores are listed first

Mean values for physiologic parameters before and after each session, regardless of active or passive engagement by the patient were compared using paired t-tests in 27 patients (see Fig. 3) and no statistically significant differences were observed across parameters. In order to evaluate differences in physiologic parameters before and after sessions and differentiated by active cycling by the patient versus passive cycling paired t-tests of mean differences were used (see Fig. 4a and b). Comparisons for patients tolerating clamping of the EVD versus continuous drainage were performed and no clinically significant differences were observed (see Fig. 5a and b).

\section{Discussion}

We found that bed-bound NCCU patients with EVDs were able to complete in-bed $\mathrm{CE}$ without differences in physiologic or neurologic parameters before and after sessions, regardless of whether the activity was passive or active. The CE was accomplished safely, without any unplanned interruption due to device dislodgement or other complication.

Recent studies support the safety and feasibility of progressive mobility from in-bed activity to out of bed

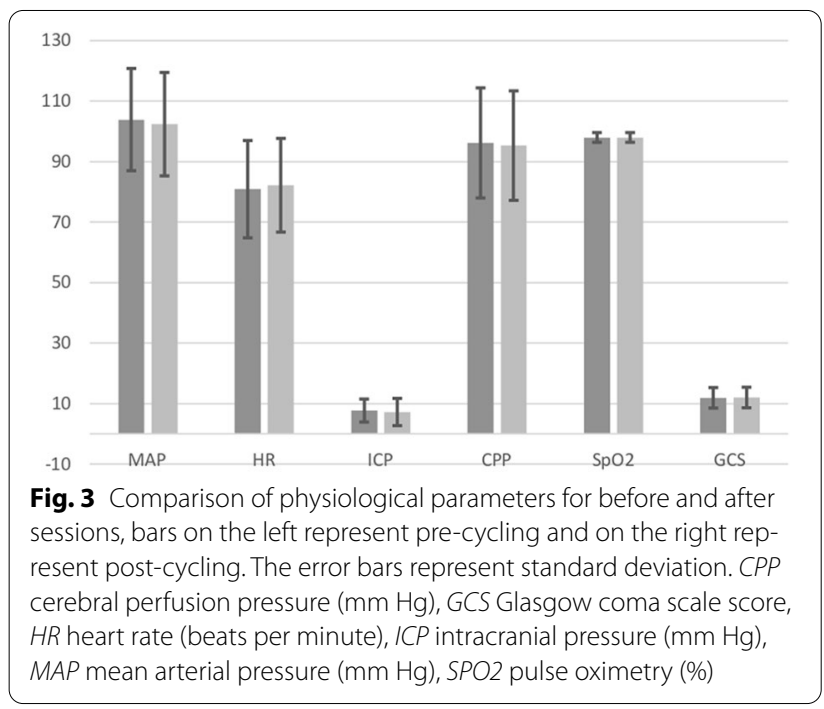

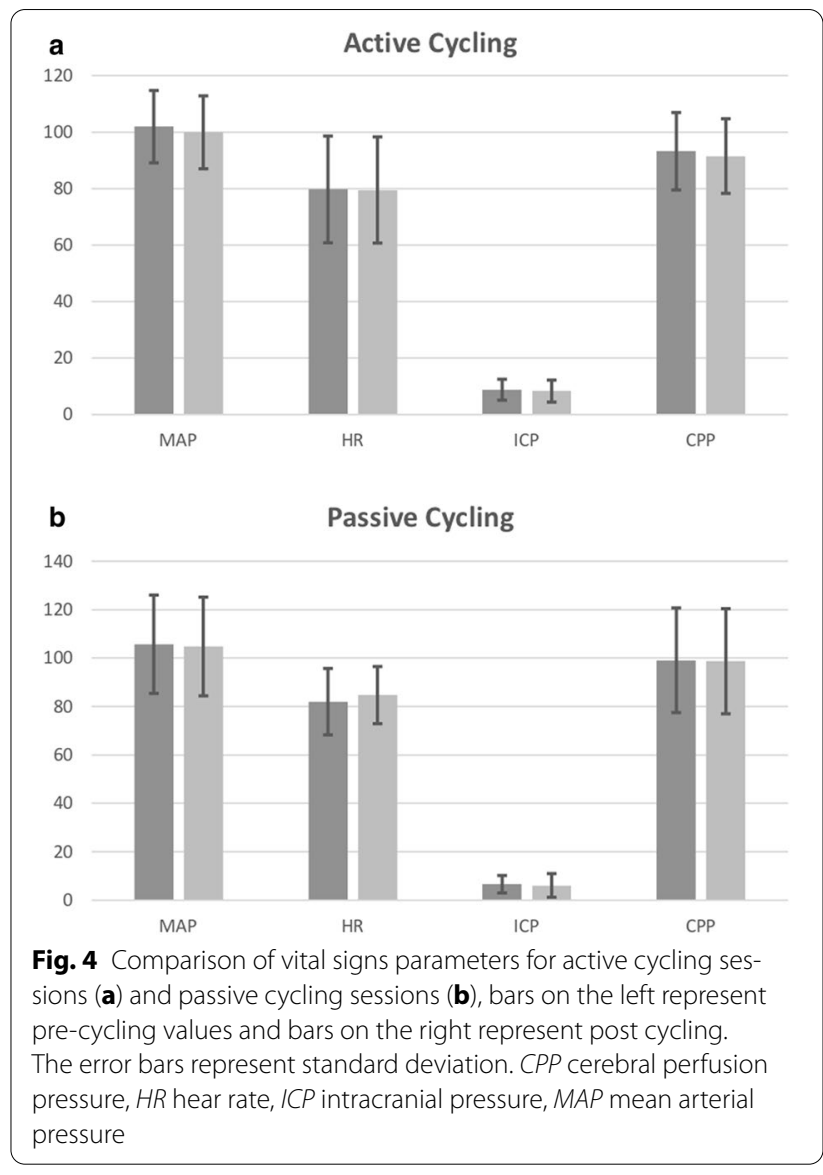

and walking in NCCU patients with external ventricular drainage (EVD) [14-16]. In nearly all reports, progressive mobility interventions begin at the time that patients demonstrate arousal, awareness and are able to follow motor commands, resulting in significant delays until mobility interventions can be implemented, if at all [1719]. Our current study adds to this literature, now with a cohort of patient who performed in-bed activity. In-bed exercises such as CE may represent a valuable alternative for these patients.

Systematic implementation of early mobility in the ICU is a key component of the ABCDEF bundle (Awakening (daily reduction of sedating medications), Breathing (ventilatory weaning), Communication, Delirium screening, Early mobility), an interdisciplinary, multimodal approach to improving outcomes of critically ill patients [20, 21]. Early progressive mobility has been associated with better functional outcomes at discharge, decreased ventilator associated pneumonia (VAP), increased ventilator free days and decreased ICU and hospital length of stay [22]. Many of these algorithms begin with a screening tool that requires patients to follow simple commands, excluding 


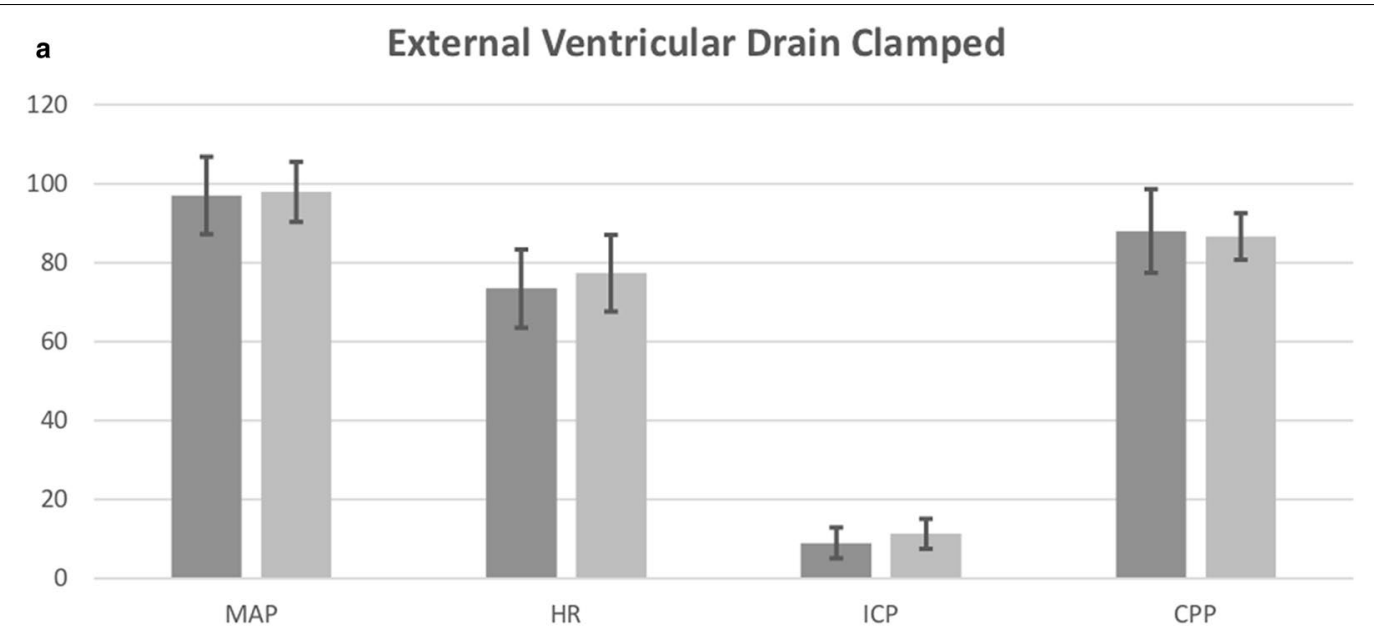

b External Ventricular Drain Unclamped

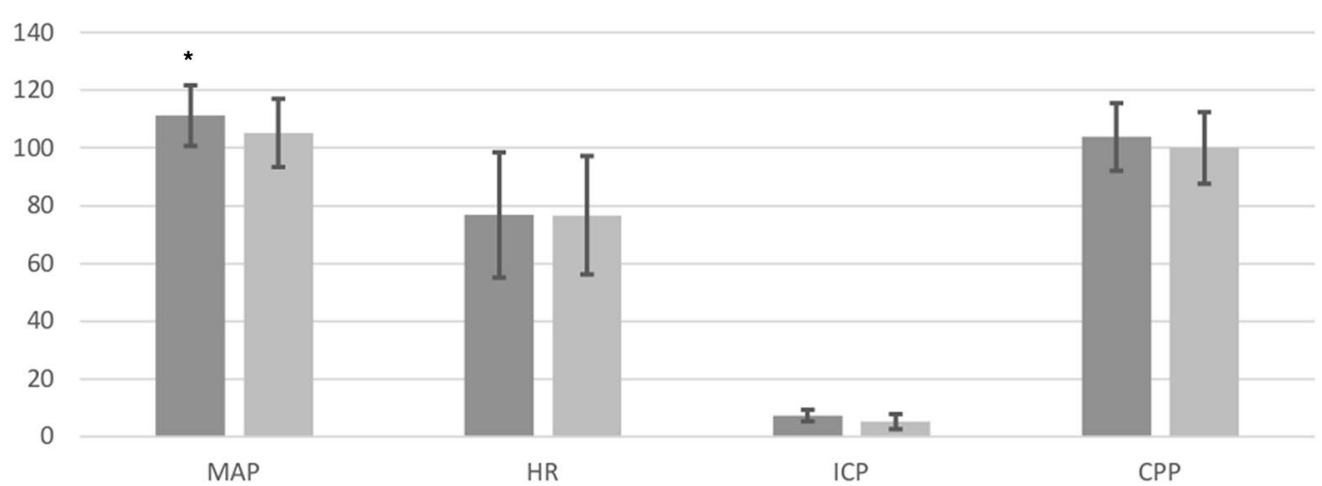

Fig. 5 Comparison of vital signs parameters for patients in whom the external ventricular drain was clamped or not draining and draining with intermittent clamping for ICP measurement. Bars on the left represent pre-cycling values and bars on the right represent post-cycling. The error bars represent standard deviation. CPP cerebral perfusion pressure, HR heart rate, ICP intracranial pressure, MAP mean arterial pressure, * ${ }^{*}$ tatistically significant difference, $P \leq 0.003$

a significant proportion of patients with impaired consciousness who may still benefit from rehabilitation therapy. Since the impact of mobilization efforts particularly in patients with disorders of consciousness is not well described, excluding these patients from early progressive mobility activities on the basis of level of consciousness could put them at a disadvantage. An activity that can be introduced early in the course of NCCU patients and that does not depend on arousal and awareness could play an important role until more sophisticated rehabilitative techniques can be added as the patient progresses. Cycle ergometry may extend the ability of clinicians to provide manual range of motion activities, increasing repetitions and allowing for simultaneous bilateral activity.

Several additional observations in this study are worth noting. We observed no significant change in heart rate in this cohort of patients, when one would have expected an exercise-associated increase in heart rate. This may suggest that patients did not achieve an aerobic threshold during these exercise sessions. Other potential explanations include use of beta-blocking medications, underlying autonomic dysfunction common to this population, and the fact that achieving maximum aerobic capacity was not the focus of the sessions. Oxygenation as measured continuously by pulse oximetry changed very little during cycling sessions and no patient required an increase in delivered oxygen. Last, the effect of passive versus active exercise on the physiology and outcomes of critically ill is not well understood. Active exercise enhances muscle bulk or strength; however passive exercise initiated early in the acute or critical care course may mitigate muscle loss, spasticity and contractures. 


\section{Limitations and Strengths}

Limitations of the study include the sample size and the cross-sectional design. A longitudinal assessment of patients receiving iterative in-bed CE during the course of their stay would provide stronger insights on the tolerability safety and efficacy of this technique during critical illness. Coupled with the appropriate sample size, a longitudinal study design could also evaluate the potential effects of CE on clinical outcomes. Another limitation is that team members did not report reasons for different session durations. As a result it is unknown if did not tolerate the cycling after a certain period of time, as reported in a previous study, or if the session duration was deliberate [5]. Strengths of this study include the variety of neuroscience patients included and the evaluation of patients with differing dependence of CSF drainage. Observations of these two factors have not been previously reported to our knowledge and the application of a mobility activity in patients requiring continuous CSF drainage suggests a novel application for in-bed CE.

\section{Conclusion}

In-bed CE performed in NCCU patients with EVDs can be accomplished safely without disruption in physiologic or neurologic parameters. Additional work is needed to determine the optimal timing, dose and the impact of this activity on neurological impairments and functional outcome in critically ill neurological patients.

\section{Author details \\ ${ }^{1}$ Department of Neurosciences Nursing, The Johns Hopkins Hospital, 1800 Orleans Street, Zayed 3 West, Room 3074, Baltimore, MD 21287, USA. ${ }^{2}$ Depart- ment of Physical Medicine and Rehabilitation, Johns Hopkins Hospital, Balti- more, MD, USA. ${ }^{3}$ Cerebrovascular Center, Cleveland Clinic, Cleveland, OH, USA. ${ }^{4}$ Department of Anesthesiology and Critical Care Medicine, Johns Hopkins University School of Medicine, Baltimore, MD, USA. ${ }^{5}$ Department of Neurol- ogy, Johns Hopkins School of Medicine, Baltimore, MD, USA.}

\section{Acknowledgements}

The authors would like to acknowledge the physical therapists and nurses working in the neurocritical care unit for their support of this work. This work was supported by efforts of the Sheikh Khalifa Stroke Institute at the Johns Hopkins Hospital. This manuscript has not been published elsewhere and is not currently under review by another publication. This work was acknowledged by the Johns Hopkins Institutional Review Board.

\section{Authors' Contribution}

The final draft of this manuscript was approved by all authors. The authors contributed in the following manner: study design and rationale (EKZ, SK, RDS), development of first draft (EKZ, SK, MNB), review and substantive contributions to the development of subsequent drafts (PG, MB, RDS), data analysis, consultation and review of methods and results sections (MB).

\section{Conflict of interest}

Conflict of interest disclosures have been completed by each author and no author had anything to disclose.

\section{Publisher's Note}

Springer Nature remains neutral with regard to jurisdictional claims in published maps and institutional affiliations.

Received: 1 July 2020 Accepted: 30 January 2021

Published online: 22 March 2021

\section{References}

1. Kumble S, Zink EK, Burch M, Deluzio S, Stevens RD, Bahouth MN. Physiological effects of early incremental mobilization of a patient with acute intracerebral and intraventricular hemorrhage requiring dual external ventricular drainage. J Neuro Care. 2017;27(1):115-9.

2. Bahouth MN, Power MC, Zink EK, Kozeniewski K, Kumble S, Deluzio S, Urrutia VC, Stevens RD. Safety and feasibility of a neuroscience critical care program to mobilize patients with primary intracerebral hemorrhage. Arch Phys Med Rehabil. 2018;99(6):1220-5.

3. Yataco RA, Arnold SM, Brown SM, David Freeman W, Carmen Cononie C, Heckman MG, Partridge LW, Stucky CM, Mellon LN, Birst JL, Daron KL, Zapata-Cooper MH, Schudlich DM. Early progressive mobilization of patients with external ventricular drains: safety and feasibility. J Neuro Care. 2018. https://doi.org/10.1007/s12028-018-0632-7.

4. Deluzio S, Vora I, Kumble S, Zink EK, Stevens RD, Bahouth MN. Feasibility of early, motor-assisted cycle ergometry in critically III neurological patients with upper limb weakness and variable cognitive status: a case series. Am J Phys Med Rehabil. 2018;97(5):e37-41.

5. Thelandersson A, Nellgard B, Ricksten SE, Cider A. Effects of early bedside cycle exercise on intracranial pressure and systemic hemodynamics in critically ill patients in a neurointensive care unit. J Neuro Care. 2016;25(3):434-9.

6. Kho ME, Martin RA, Toonstra AL, Zanni JM, Mantheiy EC, Nelliot A, Needham DM. Feasibility and safety of in-bed cycling for physical rehabilitation in the intensive care unit. J Crit Care. 2017;30(6):1419. https://doi.org/10. 1016/j.jcrc.2015.07.025

7. Kimawi I, Lamberjack B, Nelliot A, et al. Safety and feasibility of a protocolized approach to in-bed cycling exercise in the intensive care unit: quality improvement project. Phys Therapy. 2017;97:5. https://doi.org/10.1093/ ptj/pzx034.

8. Burtin C, Clerckx B, Robbeets C, et al. Early exercise in critically ill patients enhances short-term functional recovery. Crit Care Med. 2009:37(9):2499_ 505. https://doi.org/10.1097/CCM.0b013e3181a38937.

9. Pires-Neto RC, Kawaguchi YMF, Hirota AS, Fu C, Tanaka C, Caruso P, Park $M$, Carvalho CRR. Very early passive cycling exercise in mechanically ventilated critically ill patients: physiological and safety aspects a case series. PlosOne. 2013;8(9):e74182. https://doi.org/10.1371/journal.pone.0074182.

10. Machado S, Pires-Neto RC, Carvalho MTX, Soares JC, Cardoso DM, Albuquerque IM. Effects that passive cycling exercise have on muscle strength, duration of mechanical ventilation, and length of hospital stay in critically ill patients: a randomized clinical trial. J Pulmonol. 2017:43(2):134-9.

11. Perme C, Nalty T, Winkelman C, Kenji Nawa R, Masud F. Safety and efficacy of mobility interventions in patients with femoral catheters in the ICU: a prospective observational study. Cardiopulm Phys Ther J. 2013;24(2):12-7.

12. Damluji A, Zanni JM, Mantheiy E, Colantuoni E, Kho M, Needham DM. Safety and feasibility of femoral catheters during physical rehabilitation in the intensive care unit. J Crit Care. 2013;28(4):535.e9-15.

13. Boyd J, Paratz J, Tronstad O, Caruana L, Walsh J. Exercise is feasible in patients receiving vasoactive medication in a cardiac surgical intensive care unit: a prospective observational study. Aust Crit Care. 2020;33:244-9.

14. Shah SO, Kraft J, Ankam N, Bu P, Stout K, Melnyk S, Rincon F, Athar MK. Early ambulation in patients with external ventricular drains; results of a quality improvement project. J Intensive Care Med. 2018;33(6):370-4.

15. Moyer M, Young B, Wilensky EM, Borst J, Pino W, Hart M, LoBreglio J, Zaleski D, Leonor I, Kung D, Smith M, Zager E, Grady MS, Kumar M. Implementation of an early mobility pathway in neurointensive care unit patients with external ventricular devices. J Neurosci Nurs. 2017:49(2):102-7 
16. Young B, Moyer M, Pino W, Kung D, Zager E, Kumar MA. Safety and feasibility of early mobilization in patients with subarachnoid hemorrhage and external ventricular drain. J Neurocrit Care. 2019;31(1):88-96. https:// doi.org/10.1007/s12028-019-00670-2.

17. Titsworth WL, Hester J, Correia T, Reed R, Guin P, Archibald L, Mocco J. The effect of increased mobility on morbidity in the neurointensive care unit. J Neurosurg. 2012;116:1379-88.

18. Klein K, Mulkey M, Bena JF, Albert NM. Clinical and psychological effects of early mobilization in patients treated in neurologic ICU: a comparative study. Crit Care Med. 2015;43:865-73.

19. Kocan MJ, Lietz H. Special Considerations for monitoring patients in the neurointensive care unit. Crit Care Nurs Q. 2013;36(1):50-5.
20. Pandharipande P, Banerjee A, McGrane S, Ely EW. Liberation and animation for ventilated ICU patients: the ABCDE bundle for the back-end of critical care. Crit Care. 2010;14(3):157.

21. Marra A, Ely EW, Pandharipande PP, Patel MB. The ABCDEF bundle in critical care. Crit Care Clin. 2017;33(2):225-43.

22. Pun BT, Balas MC, Barnes-Daly MA, Thompson JL, Aldrich JM, Barr J, Byrum D, Carson SS, Devlin JW, Engel HJ, Esbrook CL, Hargett KD, Harmon L, Hielsberg C, Jackson JC, Kelly TL, Kumar V, Millner L, Morse A, Perme CS, Posa PJ, Puntillo KA, Schweickert WD, Stollings JL, Tan A, D'Agostino McGowan L, Ely EW. Caring for critically ill patients with the ABCDEF bundle: results of the ICU liberation collaborative in over 15,000 adults. Crit Care Med. 2019;47(1):3-14. 